\title{
Ezrin regulated myoblast differentiation/fusion and muscle fiber specialization through PKA-NFAT- MyoD/MEF2csignalling pathway
}

\section{Ruo-nan Zhang}

Hubei University of Medicine

\section{Yan Wang}

Hubei University of Medicine

Yun Liu

Hubei University of Medicine

\section{Xin Bao}

Hubei University of Medicine

\section{Wei Xu}

Hubei University of Medicine

\section{Lu-yuan Yao}

Hubei University of Medicine

\section{Magdaleena Naemi Mbadhi}

Hubei University of Medicine

\section{Long Chen}

Hubei University of Medicine

Chang-qing Hu

Hubei University of Medicine

Hong-tao Zheng

Hubei University of Medicine

\section{Zhi-feng Zhang}

Hubei University of Medicine

Jing-xuan Zhang

Hubei University of Medicine

\section{Yan Wu}

Hubei University of Medicine

\section{Yan-xia Lv}

Hubei University of Medicine

\section{Shi-You Chen}

University of Missouri

Shan Li 
Hubei University of Medicine

Shao-juan Chen

Hubei University of Medicine

Zi-jing Ren

Xiangyang Hospital, Hubei University of Medicine

Liu-liu Shi

Hubei University of Medicine

Jun-Ming Tang ( $\nabla$ tangjm416@163.com )

Hubei University of Medicine https://orcid.org/0000-0002-1591-7626

\section{Research}

Keywords: Ezrin, Differentiation, myoblast fusion, PKA, NFAT, MEF2c

Posted Date: December 31st, 2020

DOl: https://doi.org/10.21203/rs.3.rs-136524/v1

License: (9) This work is licensed under a Creative Commons Attribution 4.0 International License. Read Full License 


\section{Abstract}

Backgorund:Neuromuscular diseases are a kind of nervous system diseases that have a high disability rate.Ezrin' role in skeletal muscle has not been identified. This study aims to confirm the effect and mechanism of Ezrin on myoblast differentiation and fusion, myotube size, and myofiber type.

Method:By using immunoassaying and western blot analyses, Ezrin, MyHC,MEF2c, MyoG, PKAa/ $\beta / \mathrm{Y}, \mathrm{PKA}$ reg la, PKA reg IIßand NFATc1-c4 were detected in myoblast cells treated with Ad-Ezrin or Ad-shEzrin. Realtime PCR were used to evaluate MyoD, Myf5, MyHC-I, MyHC-Ila/b and MyHC-Ilx in myoblast cells. PKA inhibitor $\mathrm{H}-89$ or PKAreg I activator N6-Bz-cAMP were added into medium to confirm their relationship between Ezrin and PKA during myoblast differentiation/fusion. In vitro, Ad-NFATc1/c2 or Ad-shNFATc3/c4 were respectively transfected into $\mathrm{C} 2 \mathrm{C} 12$ cells, myoblast differentiation/fusion, myotube size and myofiber type were assessed by using immunostaining of MyHC, MEF2c and MyoG. In vivo, transfection of Ad-Ezrin into gastrocnemius and soleus muscles for 7 days, the numbers of MyHC-1 postivemyofibers were analyzed after immunostaining of MyHC-1.

Results: Ezrin expression were time-dependently increased during myoblast differentiation/fusion. Knockdown of Ezrin by shRNA delayed myoblast differentiation and fusion in a time dose-dependent pattern, as shown by immunostaining of MyHC. Conversely, over-expression of Ezrin by adenovirus timeand dosage-dependently promoted myoblastdifferentiation/fusion, and muscle fiber specialization characterized by increased $\mathrm{MyHC}$ I and MyHClla/b. Forced expression of Ezrin did not alter PKA, and PKAreg II a levels, but altered the levels of PKAreg I $\alpha / \beta$, Myf5 and MyoD, and leading to the accumulation of MyoG+/MEF2c+ nuclei. By contrast, Ezrin knockdown significantly decreased the PKA reg I/II ratio and MyoG+/MEF2c+ nuclei. The PKA inhibitor $\mathrm{H}-89$ remarkably abolished the beneficial effect of overexpressingEzrin on the numbers of $\mathrm{MyHC}+$ myotubes and MyoG+/MEF2c nuclei. These opposite changes mediated by knocking down Ezrin were almost eliminated by PKAreg I activator $\mathrm{N}^{6}$-Bz-cAMP. Furthermore, over-expression of NFATc2 or knockdown of NFATc4reversed the inhibitory effect of Ezrin knockdown on myoblast differentiation/fusion, resulting in the recovery of the numbers ofMyoG+/MEF2c+ nucleiin3nuclei ${ }^{+}$myotubes. Meanwhile, overexpression of Ezrin specifically induced type I muscle fiber specialization, which was associated with increased levels of NFATc1/c2. Furthermore, in vivo transfection of Ad-Ezrin into gastrocnemius and soleus muscles increased the numbers of MyHC-1 postivemyofibers. By contrast, knockdown of NFATc4resulted in the recovery to normal levels of MyHC-2b in Ezrin-knockdown myoblast cells, attributingtoregainingMyoDand MEF2c expression.

Conclusions: Ezrin trigger myoblast differentiation and fusion, myotube size, and alters muscle fiber specialization through PKA-NFAT-MyoD/MEF2C signalling pathway.

\section{Introduction}

Neuromuscular diseases are a kind of nervous system diseases that have a high disability rate, causing long-term mental and economic burden to the society, family, and the individual ${ }^{1}$. Muscle biopsy 
pathology is the most effective for the diagnosis of myopathy. Studies found that the pathological basis of muscular atrophy and muscle weakness is the degeneration and necrosis of muscle fibers. Skeletal muscles have the ability to regenerate to prevent the loss of muscle mass and maintain normal shape and function ${ }^{2}$. It is, therefore, crucial to study the characteristics of muscle fiber regeneration and their associatedfactors for the prevention and cure of neuromuscular diseases ${ }^{3}$.

Muscle satellite cells conduce to the need for physiological self-renewal and the repair of pathological injury ${ }^{4}$. Inflammatory reactions are one of the pathologies that result in myopathy,includingpolymyositis and dermatomyositis ${ }^{5}$. A recent study has shown that a more robust inflammatory area within the skeletal muscle demonstrated features of high Ezrin expression, a member of the Ezrin/radixin/moesin (ERM) proteins family. Published data supplied evidence that Ezrin could play a crucial role in transferring extracellular signal molecules into the skeletal proteins, activating the corresponding signal pathways, regulating the cell morphology, adhesion, phagocytosis, movement, and angiogenesis ${ }^{6-10}$. However, the role and mechanism of Ezrin in muscle satellite cells are still unclear.

Traditionally, the activated protein kinase $\mathrm{A}(\mathrm{PKA})$ has been linked to the unique phenomenon of myoblast differentiation/fusion and myotube formation, ascribing to the alteration in PKA regulatory subunit I (PKA $\mathrm{RI})$ under normal differentiation condition ${ }^{11}$. Our previous study has shown that it abated the ratio of PKA $\mathrm{RI} / \mathrm{RII}$ in myoblast cells, resulting in the postponement of myoblast differentiation and fusion ${ }^{12}$. Further evidence shows that ERM proteins act as PKA-anchoring proteins and sequester PKA close to its target proteins for their effective phosphorylation and functional regulation ${ }^{13}$. NFATs (Nuclear factor of activated T cells) activation mediated by PKA plays crucial role in myoblast differentiation and fusion, myotube size, and altered muscle fiber specialization ${ }^{4,13}$. In this study, knockdown of Ezrin by shRNA reduced the numbers of MyoG/MEF2C-positive cells and myotube number/size while decreasing the ratio of PKA RI/RII, causing the increased expression of NFATc2/c3/c4, suggesting that Ezrin triggered myoblast differentiation and fusion, myotube size, and altered muscle fiber specialization through PKANFAT-MEF2C signalling pathway.

\section{Method}

\section{C2C12 myoblast culture and differentiation induction}

C2C12 myoblast cells were inoculated in $75-\mathrm{cm}^{2}$ culture dishesand cultured with proliferation medium (PM) containing high glucose DMEM (HG-DMEM) supplemented with $10 \% \mathrm{FBS}$ at $37^{\circ} \mathrm{Cand} 5 \% \mathrm{CO}_{2}$. When the confluence of the cells reached $75 \%$, the PM was replaced with differentiation medium (MD) containing HG-DMEM supplemented with $2 \%$ horse serum (HS) to induce $\mathrm{C} 2 \mathrm{C} 12$ myoblast cell differentiation. Traits of myotube formation from myoblast differentiation were observed daily under a microscope ${ }^{14}$.

\section{Overexpression or Knockdown of Ezrin in vitro}


Construction of Ezrin overexpression and short hairpin RNA (shRNA) adenoviral vector were prepared as previously described ${ }^{15}$. To confirm the role of Ezrin on myoblast cells,Ad-Null, Ad-shEzrin, or Ad-Ezrin ( $1 \times$ $10^{9} \mathrm{pfu}$ ) were added into the corresponding culture dishesa day before adding the differentiation medium. These cells were then replaced with the differentiation medium for further observation.

\section{Overexpression or Knockdown of NFATs in vitro}

Construction of NFATc1/c2 overexpression adenoviral vector were prepared as previously described ${ }^{15}$. The gene accession number of overexpressing-NFATc1/c2 is NM_172390 and NM_173091, respectively. Construction of NFATc3/c4 short hairpin RNA (shRNA) adenoviral vector were prepared as previously described $^{15}$. These overexpression adenoviral vectors containing Ad-NFATc1, Ad-NFATc2, Ad-shNFATc3 and Ad-shNFATc4were obtained from Vigenebio.To confirm the role of NFATc3 or NFAtc4 on myoblast cells, the addition of Ad-shCtrl, Ad-shNFATc3, or Ad-shNFATc4 $\left(1 \times 10^{9} \mathrm{pfu}\right)$ into correspondingculture dish one day before the ISO was performed. And then these cells were replaced with differentiation medium for further observation.

\section{In vivo transfection}

For the transfection ofadult muscles, C57BL/ 6 mice were anesthetized by using anisoflurane vaporizer maintained at $2 \%$ isoflurane, $1 \mathrm{~L} / \mathrm{m}$ oxygen.Gastrocnemius and soleus muscles were exposed and injected with Ad-Ezrin $\left(1 \times 10^{10} \mathrm{pfu} \text {, two point, } 50 \mu \mathrm{m} / \mathrm{each}\right)^{15}$. Muscles were removed 7 days after transfection, frozen inisopentane cooled in liquid nitrogen, and stored at $-80^{\circ} \mathrm{C}$.

\section{Immunofluorescence Staining}

C2C12 myoblast differentiotion was determined by immunofluorescence staining. Primary monoclonal and polyclonal antibodies against MEF2C (\#5030s, 1:200, CST), MyHC (sc-20641, 1:150, Santa Cruze) were added into each well in every group and incubated for $12 \mathrm{~h}$ at $4{ }^{\circ} \mathrm{C}$. The cells were washed with PBS 3 times for $15 \mathrm{~min}$ and incubated with appropriative fluorescent dye-labeled secondary antibodies (Jackson Lab, 1:500, USA) at $25^{\circ} \mathrm{C}$ for $2 \mathrm{~h}$. The nuclei were stained with DAPI (Molecular Probes). The images for each group were photographed under Nikon 80i fluorescence microscope ${ }^{16}$.

\section{Myoblast Differentiation}

After myoblast cells were treated under DM for the indicated time, the differentiated myoblast cells were stained for MyoG or MEF2C using the primary polyclonal antibody MyoG (sc-12732, 1:150, Santa Cruze) or MEF2C (5030S, 1:400, CST) and appropriative TRITC-labeled secondary antibody (Jackson Lab, 1:500, USA). The nuclei were stained with DAPI. C2C12 myoblast cells with only 1-2 nucleuseswithin a cellular structure were evaluated withMyoG or MEF2C staining. TheMyoG + or MEF2C + cells were defined as the differentiated cells that did not fuse to form myotubes. Myoblast cells with 3 or morenucleusesin the structure of a cell were defined as myotubes. The number of double-positive nuclei under high power field $(\mathrm{HPF}, 50 \mu \mathrm{m})$ were analyzed afterdouble staining of MyoG/DAPI or MEF2C/DAPI. Two individuals who 
did not know the results evaluated the images using Image $\mathrm{J}$ (Java) software (National Institutes of Health, USA).

\section{Myoblast Fusion And Myotube Morphology}

The differentiated myoblast cells were stained for $\mathrm{MyHC}$ with the primary polyclonal antibody $\mathrm{MyHC}$ (rabbit anti-mice antibody, sc-20641, 1:150, Santa Cruze) and appropriative TRITC or FITC-labeled secondary antibody (Jackson Lab, 1:500, USA). C2C12 myoblast cells with only 1-2 nucleuses within a cellular structure were evaluated by $\mathrm{MyHC}$ staining, indicating that the $\mathrm{MyHC}+$ cells were defined as the differentiated cells without mutually fusing to myotube. Myoblast cells with 3 or more nucleuses in the structure of a cell were defined as myotube. The nuclei were stained with DAPI.

To analyze myotube size, we divided the cells into 2 groups, including short myotube with $3 \sim 5$ myoblast fusion and long myotube with more than 5 myoblast fusions. Morphology was assessed by myotube length, area (grouped less than $200 \mu \mathrm{m}$ and more than $200 \mu \mathrm{m}$ ), and the number of myotubes (grouped 3 $\sim 5$ nuclei or more than 5 nuclei myoblast fusion) under high-power magnification ${ }^{15,17}$. Two individuals who did not know the results evaluated the images using Image $\mathrm{J}$ (Java) software (National Institutes of Health, USA).

\section{Quantitative RT-PCR}

Total RNA from C2C12 myoblast cells was obtained using TRIzol (Invitrogen, Life Technologies) and transcribed into cDNA using the SuperScript II cDNA kit (Invitrogen, Life Technologies). Quantitative PCR was carried out using SYBR green PCR master mix (Thermo Fisher Scientific, Applied Biosystems, CN) in Real-Time PCR System (RotorGene 6000, Qiagen, Germany). The transcript levels of the gene of interest in each group were normalized to GAPDH levels ${ }^{18}$. The primers used are listed in Table 1. 
Table 1

The sequences of primers of qPCR.

\begin{tabular}{|lll|}
\hline Gene & Forward & Reverse \\
\hline MyoG & 5'-GAGACATCCCCCTATTTCTACCA-3' & 5'-GCTCAGTCCGCTCATAGCC-3' \\
\hline MyoD1 & 5'-CCACTCCGGGACATAGACTTG-3' & 5'-AAAAGCGCAGGTCTGGTGAG-3' \\
\hline MyHC1 & 5'-CAAGCAGCAGTTGGATGAGCGACT-3' & 5'-TCCTCCAGCTCCTCGATGCGT-3' \\
\hline MyHC2a & 5'-AGAGGACGACTGCAGACCGAAT-3' & 5'-GAGTGAATGCTTGCTTCCCCCTTG-3' \\
\hline MyHC2b & 5'-ACGCTTGCACACAGAGTCAG-3' & 5'-CTTGGACTCTTCCTCTAGCTGCC-3' \\
\hline MyHC2x & 5'-ACCAAGGAGGAGGAACAGCAGC-3' & 5'-GAATGCCTGTTTGCCCCTGGAG-3' \\
\hline GAPDH & 5'-ATGACTCCACTCACGGCAAA-3' & 5'-ATGATGACCCTTTTGGCTCC-3' \\
\hline $\begin{array}{l}\text { qPCRs were performed to identified satellite cell differentiation and muscle fibers traits by using the } \\
\text { specific primers of satellite cell differentiation markers including MyoD and MyoG, type I muscle fiber } \\
\text { makers like MyHC1, and type II muscle fiber makers such as MyHC2a, MyHC2b, and MyHC2X. }\end{array}$ \\
\hline
\end{tabular}

\section{Western Blot}

C2C12 myoblast cells were homogenized on ice in $0.1 \%$ Tween-20 homogenization buffer containing protease inhibitors. Nuclear and cytosolic protein were separated and collected using NE-PER Nuclear and Cytoplasmic Extraction Reagents according to the manufacture's instruction (78835, Thermo Fisher Scientific, USA). $20 \mu \mathrm{g}$ of protein in each well were separated by 7 or $10 \%$ SDS-PAGE gel electrophoresis and transferred onto PVDF membrane (Millipore). After blocking with $5 \%$ nonfat milk, the membranes were incubated with primary antibodies, including a-tubulin (T9026, 1:5000, Sigma), Histone H3 (ab6002, 1:500, ABCAM), NFATc1 (ab2796, 1:500, Abcam), NFATc2 (ab2722,1:500, Abcam),

NFATc3(ab83832,1:500, Abcam), NFATc4 (SAB4501982, 1:1000, Sigma) and MyHC (sc-20641, sc-376157, 1:500, Santa Cruze) overnight at $4{ }^{\circ} \mathrm{C}$, respectively. Thereafter, the blots were incubated with corresponding horseradish peroxidase (HRP)-conjugated secondary antibodies (anti-rabbit lgG, anti-goat IgG, 1:10000; Santa Cruz) for 90 min. Protein expression was detected by enhanced chemiluminescence method, and the Image $\mathrm{J}$ software was used for gray value analysis ${ }^{19}$.

\section{Statistical analysis}

Data of quantitative and semi-quantitative analysis presented are mean \pm SD.Paired or unpaired Student's $t$-test determined statistical significance between the two groups.One-way ANOVA was used to compare the results for more than two experimental groups to specify the differences between groups. $P<0.05$ is considered meaningful.

\section{Results}

Ezrin is expressed in $\mathrm{C} 2 \mathrm{C} 12$ cells during myoblast differention and fusion 
To confirm the role of Ezrinin C2C12 myoblast cells, we determined the changes ofEzrin expression during myoblast differentiation and fusion by western blot. We found that the expression ofEzrin gradually increased, reaching its peak on day 4 of myoblast differentiation(Fig. 1).This expression is accompanied byC2C12 myoblast cell alterations, that time-dependently differentiated into mature muscle cells,formingmyotubes characterized by $\mathrm{MyHC}$ positive staining under differentiation medium containing $2 \%$ HS-DMEM. These results indicated that Ezrin could play an essential role in myoblast differentiation and fusion.

Our results further showed that the number of $\mathrm{MyHC}+$ myotubes with either $3-5$ or $5^{+}$nuclei increased upontreatment with Ad-Ezrin in a time-dependent manner (Fig. 1E-1H). Conversely, knockdown of Ezrin by shRNAdid not only obviously reduce $\mathrm{MyHC}+$ cell number, but it also dramatically decreasedmyotube numbers with either $3-5$ or $5^{+}$nuclei (Fig. Figure $1 \mathrm{E}-1 \mathrm{H}$ ). Thisindicates that knockdown of Ezrincan almost effectively block myoblast differentiation and fusion. Combined with Fig. 1A-1D, these results demonstrated that Ezrinis involved in early differentiation and fusion of myoblast.

\section{Transfection efficiency of overexpression or knockdown of Ezrin into myoblast cell}

To further determine the effect of Ezrinin $\mathrm{C} 2 \mathrm{C} 12$ myoblast differentiation and fusion, we transfected the C2C12 cells with an adenovirus-mediated overexpression of Ezrin, or knockdown of Ezrin by shRNA.Assessment of the transfection efficiency revealed that following the application of 100 optimal multiplication of infection (MOI) with the adenovirus, $\mathrm{C} 2 \mathrm{C} 12$ myoblast cells almost reached a confluence of $95 \%$ (sfig.1A-1C). His-tag was used to confirm the successful expression of the exogenous genes and their respective functions (sfig.1C-1E).

\section{Ezrin Altered The Muscle Fiber Types}

Indeed, there are different types of muscle fibers formed by MyHC-1, MyHC-2a, MyHC-2b, or MyHC-2X. MyHC-1-positive type I fiber shows a slim-long feature. MyHC-2a, MyHC-2b, and MyHC-2X positive type II fiber has thick-short traits ${ }^{14}$. In line with the increased myotube formation following forced-expression of Ezrin, MyHC-1 expressions were markedly increased (Fig. 2C-2F). By contrast, MyHC-2a and MyHC-2b expressions were evidently increased in knock-downing-Ezrin myoblast cells. However, MyHC-2X expressions were not obviously altered in either overexpression or knockdown of Ezrin (Fig. 2C-2F). More importantly, overexpression of Ezrin in gastrocnemius (GA) and musculi soleus (SL) increased numbers of MyHC-1postive muscle fibers(Fig. 2G-2J). Nevertheless, these results suggested that Ezrin regulated the expressions of all MyHC isoforms.

\section{MYOG/MEF2C Involved Ezrin-mediated Myoblast Differentiation And Fusion}

Since MyoG and MEF2C play a crucial role in the initiation and later of myoblast cells differentiation ${ }^{20}$, we used MyoG and MEF2C staining to confirm the relationship between Ezrin and myoblast differentiation. Our results showed that following treatment of Ad-Ezrin,the number and percentage of $\mathrm{MyoG}^{+}$nuclei were higher than that of MEF2C + nuclei within the myoblast during $\mathrm{C} 2 \mathrm{C} 12$ myoblast 
differentiation(Fig. 3A-2G). Knockdown of Ezrin by shRNA markedly reduced the percentage of MyoG + and MEF2C + nuclei cells in $\mathrm{C} 2 \mathrm{C} 12$ myoblast cells(Fig. 3A-2G).Moreover, the percentage of MEF2 $\mathrm{C}^{+}$ nuclei was lower than that of MyoG + nuclei in Ezrin-knockdownC2C12 myoblast cells(Fig. 3C-2G). These results indicated thattheknockdown of Ezrincouldinhibit the initiation and late phase of myoblast differentiation through MyoG and MEF2C, especially MyoG.

\section{Ezrin regulated myoblast differentiation and fusion through PKA signalling pathway}

Previous results have shown that PKA and PKAreg $\mathrm{I} / \mathrm{II}$ ratio play crucial roles in controlling myoblast differentiation and fusion ${ }^{11,12}$. To confirm if Ezrin's role in myoblast differentiation and fusion was involved in PKA signalling pathway, we used western blot to detect PKA signaling(Fig. 4A-4G). Our results revealed that, the overexpression of Ezrin did not alter PKAreg II levels, but it significantly increased the levels of PKAa,PKAreg la,and PKAreg I $\beta$, resulting in an increased PKAreg I/II ratio. By contrast, knockdown of Ezrin by shRNA significantly reduced PKAreg laand PKAreg Ißlevels, but it did not alterPKAreg II levels, resulting in a decreased PKAreg I/II ratio (Fig. 4A-4G).

\section{PKA Involved Ezrin-mediated Myoblast Differentiation And Fusion}

PKA activity is found to have effects on myoblast differentiation and fusion ${ }^{11,12}$. Combining with the above results that the overexpression or knockdown of Ezrinaffected myoblast differentiation and fusion by altering PKA activity, we treated $\mathrm{C} 2 \mathrm{C} 12$ cells with PKA inhibitor $(\mathrm{H}-89)$. We found that PKA inhibitor $(\mathrm{H}-$ 89)abolished the beneficial role of Ezrin-mediated C2C12 myoblast differentiation and fusion. By contrast, both PKA activator and cAMP analogues reversed the inhibitory effects of Ezrin knockdown onC2C12 myoblast differentiation and fusion (Fig. 5A-5D). These results indicated that the effect of Ezrinduring myoblast differentiation and fusion could be associated with PKA signalling.

\section{Ezrin regulated myoblast differentiation and fusion through PKA-MyoG/MEF2C signalling pathway}

Indeed, myotube is formed by the fusion of differentiated myoblasts, which is characterized by three $\left(3^{+}\right)$ or morenuclei in the structure of a cell ${ }^{15,17}$. As shown in Fig. $6 \mathrm{~A}-6 \mathrm{~J}$, we found that knockdown of Ezrin by shRNA markedly decreased the percentage of MyoG + and MEF2C + nuclei in less than 3 cells and $3^{+}$myotubes, and these effects could be abolished by PKA activator. By contrast, overexpression of Ezrin substantially increased the number of MyoG + or MEF2C + nucleus in less than 3 and $3^{+}$myotubes. However, the specific changes were almost entirely cancelled by the PKA inhibitor (Fig. 6C-6J). These results indicated that Ezrin participated in $\mathrm{C} 2 \mathrm{C} 12$ myoblast differentiation and fusion with coordination of MyoG and MEF2C.

\section{NFATs signaling involved in the regulation of myotube types mediated by Ezrin}

Previous studies have reported on two types of muscle fibers, including slow (slim-long) and fast (thickshort) myofibers, MyHC-1 forms the former, and MyHC-2a, MyHC-2b or MyHC-2X the latter ${ }^{24,25}$. Corresponding with myoblast differentiation and fusion, especially myofiber specification mediated by 
NFATs ${ }^{26-32}$. We firstly found that overexpressing Ezrin increased nucleus NFATc1/c2 levels while decreasing NFATc3/c4 levels. Conversely, knocking down Ezrin increased NFATc3/c4 levels while decreasing NFATc1/c2 levels (Fig. 7A-7E). Secondly, either NFATc2 overexpression or NFATc4 knockdown almost completely reversed the inhibitory effects of knocking-down Ezrin on myoblast differemtiation and fustion, resulting in the recovery of numbers of slim-long myofibers (nuclei numbers more than 5) (Fig. 8A). Eventually, NFATc1/c3 knockdown did not significantly recuperate the inhibitory role of knocking-down Ezrin in myoblast differemtiation/fustion (sFigure2). Meanwhile, we found that knockdown of Ezrin increased MyoD expressions, the specific effects could be abolished by AdNFATc1/c2 or Ad-shNFATc3/c4. More importantly, the specific increase in MyHC-2a and MyHC-2b mediated by knockdown of Ezrin could obviously inversed by Ad-NFATc1/c2 or Ad-shNFATc4(Fig. 8B7D),respectively.Thus, Ezrinmainly regulated myoblast differentiation/fusion, and myofiber specification through integration role of NFATc2/c4 signaling pathway.

\section{Ezrin regulated myoblast differentiation and fusion through NFATc2/c4-MyoG/MEF2C signaling pathway}

Since MyoG and MEF2Cinvolved in the ontrol of the initiation and later stage of myoblast differentiation, respectively ${ }^{26}$. As shown in Fig. $8 \mathrm{~A}$ and sFigure3A-3F, we found that knockdown of Ezrin by shRNA markedly decreased the numbers and percentage of MyoG + and MEF2C + nuclei in less than 3-nuclei cells and 3-nuclei ${ }^{+}$myotubes, and these effects could be abolished by Ad-NFATc2 or Ad-shNFATc4. In addition, Ad-NFATc1 or Ad-shNFATc3 reversed the numbers of MEF2C + nuclei in 3-nuclei ${ }^{+}$myotubes. These results indicated that Ezrin participated in $\mathrm{C} 2 \mathrm{C} 12$ myoblast differentiation and fusion with coordination of MyoG and MEF2C, which were associated with NFATc2/c4, at least in part.

\section{Discussion}

In this study, we made three novel observations. Firstly,we found that theEzrin expression has a timedynamic characteristic during myoblast differentiation and fusion.Secondly, Ezrin significantly controlled myoblast differentiation and fusion. And lastly, Ezrin regulated C2C12 myoblast differentiation, fusion, and myotube type formationviaPKA RI-NFAT-MyoD/MEF2C signalling pathway.

Ezrin belongs to the ERM family of proteins that play structural and regulatory roles in the assembly and stabilization of plasma membrane interactions through their ability to interact with transmembrane proteins and the cytoskeleton ${ }^{13}$. As one of ERM proteins, Ezrinactivated the intracellular signal pathways through transferring extracellular signal molecules into actin cytoskeleton, affecting several key cellular processes, including membrane dynamics, cell adhesion, cell survival, motility, and determination of cell shape $^{6-10}$. Indeed, these cellular processes were associated with myoblast differentiation and fusion ${ }^{21}$. Forthe first time, we found that Ezringradually increased during myoblast differentiation/fusion, and rapidly decreased when formed myotubes approached and/or reached maturity. Furthermore, overexpression of Ezrin significantly accelerated the process of myoblast differentiation and myotube formation. Conversely, this process was delayed by the knockdown ofEzrin. These results suggest that 
Ezrin could act as a crucial factor during skeletal muscle regeneration and restoration in physiological and pathological conditions.

Previous studies have shown that the overexpression of PKA inhibited myogenic differentiation, contributing to HDAC4 phosphorylation and the transcriptional repression of muscle-specific genes by the myogenic regulators Myf-5 and MyoD $22,23,24,25$. Furthermore, ERM proteins,includingEzrin, are reported toact as PKA-anchoring proteins and sequester PKA close to its target proteins for their effective phosphorylation and functional regulation ${ }^{13}$. However, our results did not show anyapparentchanges in expressions of Myf- 5 and MyoD in overexpressingEzrin in myoblast cells, but showed increased numbers of MyoGandMEF2C-positive myoblast cells and myotubes. Actually, MyoG and MEF2C play an essential role in the initiation and later stage of myoblast differentiation, respectively ${ }^{26}$. Recent reports have also associated PKA RI and RII to myoblast differentiation and myotube formation ${ }^{11,12}$. Of interest, we found that overexpressingEzrin markedly increased PKA RI levels, leading to an increased PKA RI/RII ratio, accompanied by an acceleration of myoblast differentiation and fusion. Furthermore, the knockdown of Ezrin, resulting in a lower PKA RI/RII ratio, inhibitory effects observed during myoblast differentiation/fusion could be reversed by the PKA RI activator. More importantly, PKA RI activator almost completely recovered the numbers of MyoG or MEF2C positive myotubes. Therefore, Ezrin could promotemyoblast differentiation/fusionviaPKA RI/RII-MyoG/MEF2C signalling pathway.

Existing data have shown that NFATs act as a crucial player in myoblast differentiation and fusion, especially myofiber specification ${ }^{26-32}$. Moreover, NFATs activities are frequently regulated by the PKAcalcineurinsignalling pathway during cell differentiation $33,34,35,36$. Indeed, NFATc2 primarily controlled myoblast recruitment and myoblast fusion $37,38,39,40$. Similarly, we found that the inhibitory effect of myoblast fusion by knockdown of Ezrin could be restored by the overexpression of NFATc2, but not NFATc1. In line with stimulatory effect of constitutively active NFATC1 (caNFATc1) on MyHC-1 expresison ${ }^{41}$, the increased NFATc1 levels by overexpression of Ezrin induced MyHC-1 expression. However, enhanced NFATc1 levels by Ad-Ezrin did not decrease MyHC-2b as caNFATc1 did, but keeping its normal levels. Meanwhile, Ad-Ezrin reduced NFATc3/c4 levels in myoblast cells, but maintaining MyHC-2a expression, which was different from the inhibitory role of knockdown for NFATc3 and NFATc4 in the expression of MyHC-2a ${ }^{30}$. These different changes could be related with the increased levels of NFATC2 by Ad-Ezrin, because NFATc2 acted as important role in MyHC-2a and MyHC-2X expressions ${ }^{37,40,41}$. In a word, altered NFATs signaling by Ezrinaffectedmyoblast differentiation/fusion, especiallytype- 1 and type2amuscle fiber specialization.

In relation to muscle fiber type specialization, slow muscle specialization and fast to slow myofiber-type switch need the coordination of MEF2C and MyoD $39,40,41,42,43$ respectively. In this study, the overexpression ofEzrinincreased the MEF2C levels within the nucleus while decreasingthelevels of MyoD in myoblast cells, resulting in the increased MyHC-1 expressions, accompanied by normal levels of MyHC$2 \mathrm{a}, \mathrm{MyHC}-2 \mathrm{~b}$ and MyHC-2X. The knockdown of Ezrinreduced MEF2C + myoblast cells numbers while increasing MyoDexpressions, leading to special changes that the levels MyHC-2a and MyHC-2b were 
obviously increased. Previous reports have shown that NFATc2 could perform specific role in MyHC-2a and MyHC-2X expression with synergistic effect of MyoD while NFATc1 promoted MyHC-1 expression through inhibiting MyoD signaling ${ }^{37,40}$. More, NFATc4 has shown the inhibitory role in myoblast fusion ${ }^{38}$, and appears to mostly contribute to fast muscle fiber formation, especially MyHC- $2 b^{30}$. The overexpression of NFATc4 affects the differentiation process by decreasing the expression of late differentiation markers, including MEF2c, and impairs myotube formation ${ }^{30,38}$. We found that these similar effects mediated by the knockdown of Ezrin could be reversed by knockdown of NFATc4. Thus, Ezrincould regulate myoblast fusion and type Ilb muscle fiber specialization through NFATc4-MEF2C signalling pathway.

\section{Conclusion}

Ezrinaffects myoblast differentiation, fusion, and muscle fiber specialization via PKA-NFAT-MyoD/MEF2C signalling pathway.

\section{Abbreviations}

DAPI: 4',6-Diamidino-2-Phenylindole; DMEM: Dulbecco's Modified Eagle's Medium; FBS: Fetal Bovine Serum; HF: heart failure; HRP: horseradish peroxidase;HS: horse serum; IF: Immunofluorescence; MD: Muscular dystrophy; MyHC: myosin heavy chain; NFAT:nuclear factor of activated T cells; MEF2C:Myocyte-specific enhancer factor 2C; MyoD: myogenic differentiation 1; M: Mol/L; PKA: protein kinase A; PKA RIa: PKA regulatory subunit Ia; PVDF: polyvinylidene fluoride.

\section{Declarations}

\section{Ethics approval and consent to participate}

Not applicable.

\section{Competing interests}

The authors declare that they have no competing interests.

\section{Availability of Data and material}

Please contact corresponding author for data requests.

\section{Consent for publication}

Not applicable.

\section{Author contributions}


R.L.Zmainly performed cells experimental and prepared the first draft; L.X and C.Q.H fulfilled qPCR; H.T.Z and X.Bperformed protein detection; Y.W and Z.F.Zparticipated in the immunostaining; S. L, Z.J.Rand X.W participated in myotube analysis; L.Y.Y andL.Cparticipated in Adenovirus preparation; J.X.Z and Y.Whad a hand in the study design; M.N.M andS.Y.C partook in revising the manuscript;L.L.S and J.M.T composed the study, partook in the design and coordination of the whole study, and was conducive to revising the manuscript. All authors have read and agreed to the final draft.

\section{Funding}

The present study was supported by projects from the Foundation of Hubei Science\& Technology Department (2018ACA162 to J.M.T), Hubei Province's Outstanding Medical Academic Leader program, the Foundation of Hubei University of Medicine (HBMUPI201807, FDFR201601 to J.M.T), the National Natural Science Foundation of China (81670272 to J.M.T), Health Commission of Hubei Province scientific research project (WJ2019M051).

\section{Acknowledgements}

Not applicable

\section{References}

1.Smith SA, Downey RM, Williamson JW, Mizuno M. Autonomic dysfunction in muscular dystrophy: a theoretical framework for muscle reflex involvement. Front Physiol. 2014; 5:47.

2.Kowalski K, Dos Santos M, Maire P, Ciemerych MA, BrzoskaE.Induction of bone marrow-derived cells myogenic identity by their interactions with the satellite cell niche.Stem Cell Res Ther. 2018;9(1):258.

3.Sun C, Serra C, Lee G, Wagner KR.Stem cell-based therapies for Duchenne muscular dystrophy.Exp Neurol. 2020;323:113086.

4.Yin H, Price F, Rudnicki MA. Satellite cells and the muscle stem cell niche.Physiol Rev. 2013;93(1):23-67.

5.DeWane ME, Waldman R, Lu J. Dermatomyositis: Clinical features and pathogenesis.J Am AcadDermatol. 2020;82(2):267-281.

6.Fehon RG, McClatchey Al, Bretscher A. Organizing the cell cortex: the role of ERM proteins. Nat Rev Mol Cell Biol. 2010;11(4):276-87.

7.Pore D, Gupta N.Ezrin-radixin-moesin family proteins in the regulation of B cell immune response. Crit Rev Immunol. 2015,35:15-31.

8.Bretscher A, Edwards K, Fehon RG. ERM proteins and merlin: integrators at the cell cortex. Nat Rev Mol Cell Biol. 2002;3:586-599. 
9.Arpin M, Chirivino D, Naba A, Zwaenepoell.Emerging role for ERM proteins in cell adhesion and migration. Cell AdhesMigr.2011;5:199-206.

10.Kishore R, Qin G, Luedemann C, Bord E, Hanley A, Silver M, Gavin M, Yoon YS, Goukassian D, Losordo DW. The cytoskeletal protein ezrin regulates EC proliferation and angiogenesis via TNF-alpha-induced transcriptional repression of cyclin A. J Clin Invest. 2005;115(7):1785-96.

11. Han SY, Park DY, Lee GH, Park SD, Hong SH. Involvement of type I protein kinase A in the differentiation of L6 myoblast in conjunction with phosphatidylinositol 3-kinase. Mol Cells. 2002;14(1):68-74.

12.Chen SJ, Yue J, Zhang JX, Jiang M, Hu TQ, Leng WD, Xiang L, Li XY, Zhang L, Zheng F, Yuan Y, Guo LY, Pan YM, Yan YW, Wang JN, Chen SY, Tang JM.Continuous exposure of isoprenaline inhibits myoblast differentiation and fusion through PKA/ERK1/2-FOXO1 signaling pathway.Stem Cell Res

Ther.2019;10:70.

13.Kawaguchi K, Yoshida S, Hatano R, et al. Pathophysiological Roles of Ezrin/Radixin/Moesin Proteins. Biological \& Pharmaceutical Bulletin, 2017, 40(4):381-390.

14.Chen ShJ, Xiang Li, Jiang M, et al. Isoprenaline induced muscle atrophy by inhibiting C2C12 cell differentiation into skeletal muscle cells. Chin J Cell Biol. 2017; 39:1178-1187.

15.Hicks MR, Cao TV, StandleyPR. Biomechanical strain vehicles for fibroblast-directed skeletal myoblast differentiation and myotube functionality in a novel coculture. Am J Physiol Cell Physiol. 2014; 307:C67183.

16.Ross JA, Levy Y, Svensson K, et al.SIRT1 regulates nuclear number and domain size in skeletal muscle fibers.J Cell Physiol. 2018, 233:7157-716.

17. Horsley V, Jansen KM, Mills ST, Pavlath GK. IL-4 acts as a myoblast recruitment factor during mammalian muscle growth..Cell. 2003;113:483-94.

18.Chen X, Wan J, Yu B, et al.PIP5K1 a promotes myogenic differentiation via AKT activation and calcium release. Stem Cell Res Ther. 2018; 9(1):33.

19.Li GH, Luo B, Lv YX, et al.Dual effects of VEGF-B on activating cardiomyocytesand cardiac stem cells to protect the heart against short- and long-term ischemia-reperfusion injury.JTrans/ Med. 2016; 14:116.

20.Cheng X, Du J, Shen L, Tan Z, Jiang D, Jiang A, Li Q, Tang G, Jiang Y, Wang J, Li X, Zhang S, Zhu L. MiR-204-5p regulates C2C12 myoblast differentiation by targeting MEF2C and ERRY. Biomed Pharmacother. 2018; 101:528-535.

21.Palmer S, Groves N, Schindeler A, Yeoh T, Biben C, Wang CC, Sparrow DB, Barnett L, Jenkins NA, Copeland NG, Koentgen F, Mohun T, Harvey RP. The small muscle-specific protein Csl modifies cell shape 
and promotes myocyte fusion in an insulin-like growth factor 1-dependent manner. J Cell Biol. 2001;153(5):985-98.

22. Hardy S, Kong Y, Konieczny SF. Fibroblast growth factor inhibits MRF4activity independently of the phosphorylation status of a conserved threonineresidue within the DNA-binding domain. Mol Cell Biol. 1993;13(10):5943-56.

23.Winter B, Braun T, Arnold HH. cAMP-dependent protein kinase repressesmyogenic differentiation and the activity of the muscle-specific helix-loop-helix transcription factors Myf-5 and MyoD. J Biol Chem. 1993;268(13):9869-78.

24.Doddi SK, Kummari G, M V J, Kalle AM. Protein kinase A mediates novel serine-584 phosphorylation of HDAC4. Biochem Cell Biol. 2019;97(5):526-535.

25.Arnold HH, Braun T, Bober E, Buchberger A, Winter B, Salminen A. Regulationofmyogenin expression in normal and transformed myogenic cell lines. SympSocExp Biol. 1992;46:37-51.

26. Cho YY, Yao K, Bode AM, Bergen HR 3rd, Madden BJ, Oh SM, Ermakova S, Kang BS, Choi HS, Shim JH, Dong Z.RSK2 mediates muscle cell differentiation through regulation of NFAT3.J Biol Chem. 2007;282:8380-92.

27. O'Connor R S ,Mills S T, Jones K A, et al. A combinatorial role for NFAT5 in both myoblast migration and differentiation during skeletal muscle myogenesis. Journal of Cell Science. 2007. 120:149-159.

28.Kegley KM, Gephart J, Warren GL, PavlathGK.Altered primary myogenesis in NFATC3(-/-) mice leads to decreased muscle size in the adult.Dev Biol. 2001;232:115-26.

29.Xu M, Chen X, Chen D, Yu B, Li M, He J, Huang Z.MicroRNA-499-5p regulates skeletal myofiber specification via NFATc1/MEF2C pathway and Thrap1/MEF2C axis.Life Sci. 2018;215:236-245.

30.Calabria E, Ciciliot S, Moretti I, Garcia M, Picard A, Dyar KA, Pallafacchina G, Tothova J, Schiaffino S, MurgiaM.NFAT isoforms control activity-dependent muscle fiber type specification.Proc Natl AcadSci US A. 2009;106:13335-40.

31.Du J, Shen L, Zhang P, Tan Z, Cheng X, Luo J, Zhao X, Yang Q, Gu H, Jiang A,Ma J, Tang Q, Jin L, Shuai S, Li M, Jiang Y, Tang G, Bai L, Li X, Wang J, ZhangS, Zhu L. The regulation of skeletal muscle fiber-type composition by betaine is associated with NFATc1/MyoD. J Mol Med (Berl). 2018;96:685-700.

32.Ehlers ML, Celona B, Black BL.NFATc1 controls skeletal muscle fiber type and is a negative regulator of MyoDactivity.Cell Rep. 2014;8:1639-1648.

33.Khalilimeybodi A, Daneshmehr A, Sharif KashaniB.Ca2+-dependent calcineurin/NFAT signaling in $\beta$ adrenergic-induced cardiac hypertrophy.GenPhysiolBiophys. 2018;37:41-56. 
34.Horsley V, PavlathGK.NFAT: ubiquitous regulator of cell differentiation and adaptation.J Cell Biol. 2002;156:771-4.

35.Swoap SJ, Hunter RB, Stevenson EJ, Felton HM, Kansagra NV, Lang JM, EsserKA, KandarianSC.Thecalcineurin-NFAT pathway and muscle fiber-type gene expression.Am J Physiol Cell Physiol. 2000;279:C915-24.

36.Daou N, Lecolle S, Lefebvre S, dellaGaspera B, Charbonnier F, Chanoine C, Armand AS.A new role for the calcineurin/NFAT pathway in neonatal myosin heavy chain expression via the NFATc2/MyoD complex during mouse myogenesis. Development. 2013;140:4914-25.

37. Horsley V, Friday BB, Matteson S, Kegley KM, Gephart J, PavlathGK.Regulation of the growth of multinucleated muscle cells by an NFATC2-dependent pathway.J Cell Biol. 2001;153:329-38.

38.Perroud J, Bernheim L, Frieden M, Koenig S.Distinct roles of NFATc1 and NFATc4 in human primary myoblast differentiation and in the maintenance of reserve cells. J Cell Sci. 2017;130:3083-3093.

39. Horsley V, Jansen KM, Mills ST, Pavlath GK IL-4 acts as a myoblast recruitment factor during mammalian muscle growth. Cell. 2003;113:483-94.

40Daou N, Lecolle S, Lefebvre S, dellaGaspera B, Charbonnier F, Chanoine C, Armand AS.A new role for the calcineurin/NFAT pathway in neonatal myosin heavy chain expression via the NFATc2/MyoD complex during mouse myogenesis. Development. 2013;140:4914-25.

41.McCullagh KJ, Calabria E, Pallafacchina G, Ciciliot S, Serrano AL, Argentini C, Kalhovde JM, Lømo T, SchiaffinoS.NFAT is a nerve activity sensor in skeletal muscle and controls activity-dependent myosin switching.Proc Natl AcadSci U S A. 2004;101:10590-5.

42.Melissa L, Ehlers, Barbara. NFATc1 Controls Skeletal Muscle Fiber Type and Is a Negative Regulator of MyoD Activity. Cell Reports. 2014;8:1639-1648.

43.Koo JH, Kim TH, Park SY, Joo MS, Han CY, Choi CS, Kim SG.Ga13 ablation reprograms myofibers to oxidative phenotype and enhances whole-body metabolism. JClin Invest. 2017;127:3845-3860.

\section{Figures}



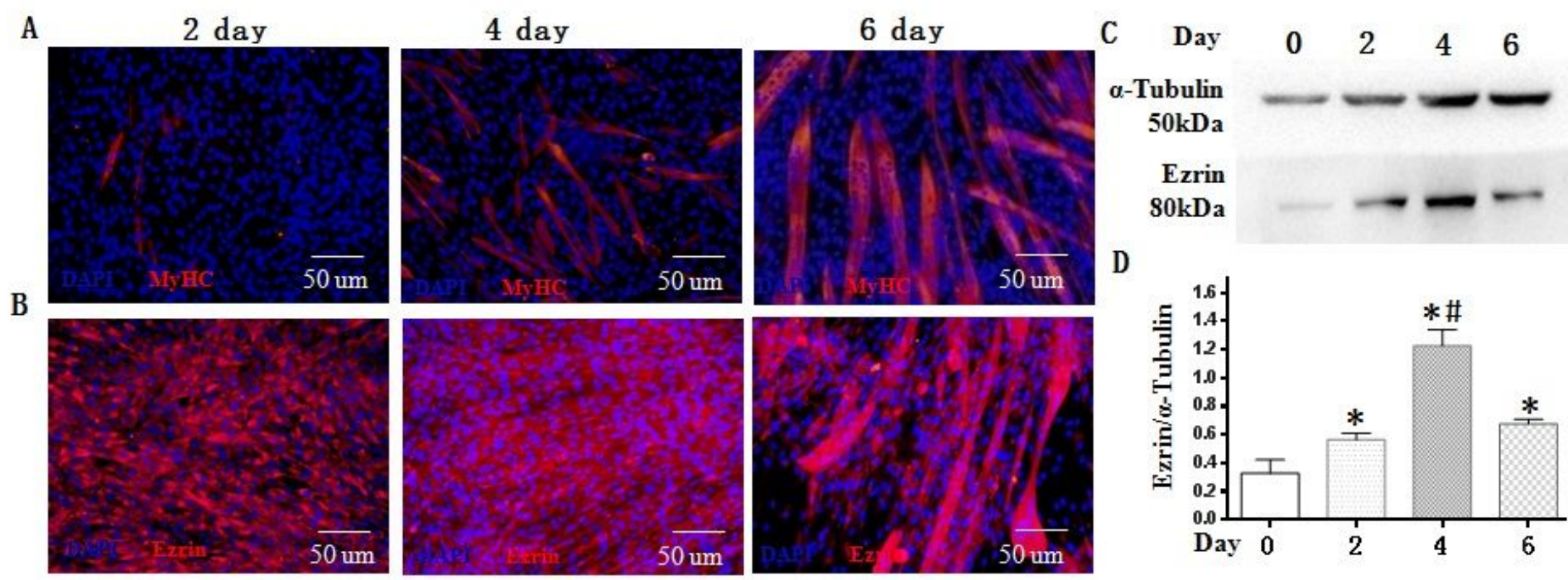

D
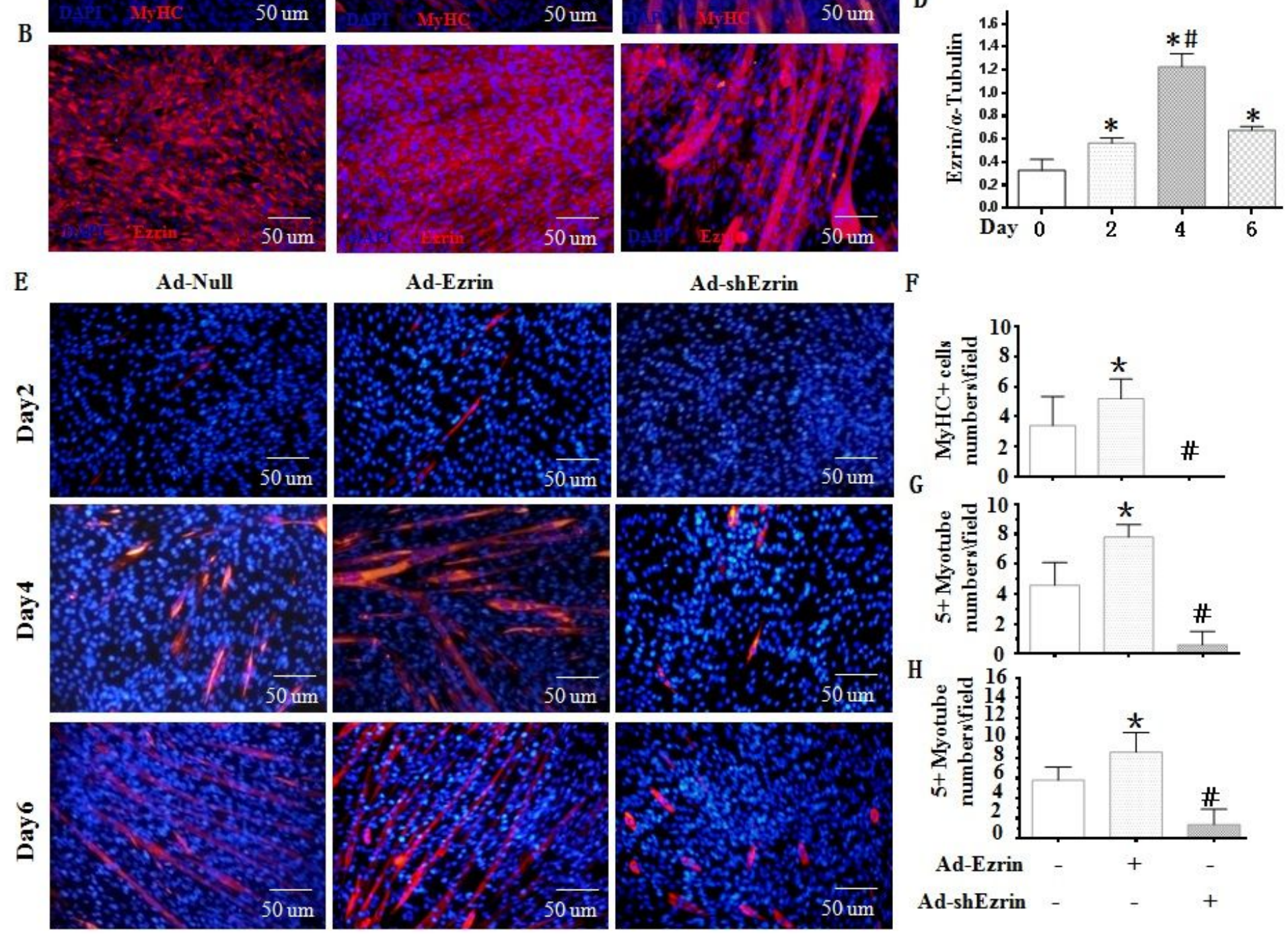

F
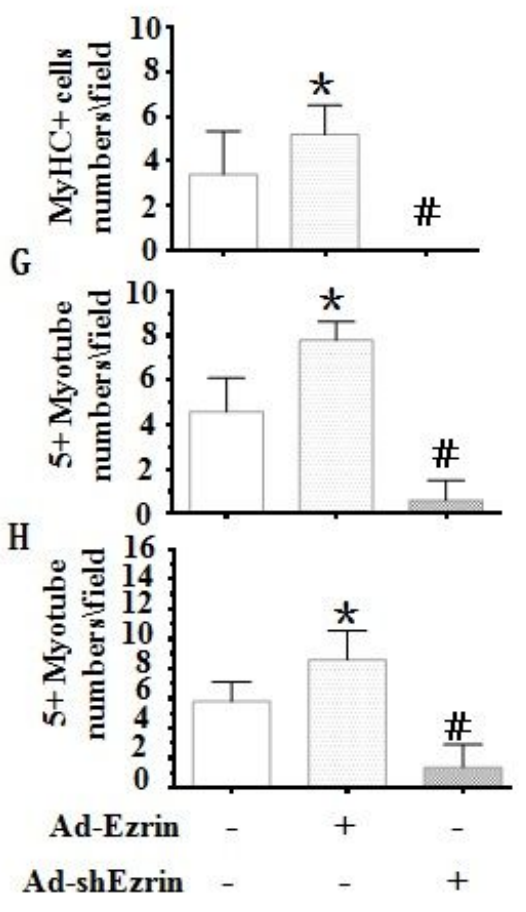

\section{Figure 1}

Time course of Ezrin during myoblast differentiation and fusion. Typical image for MyHC during myoblast differentiation and fusion.(B) Western blot for Ezrin expressions at 0, 2,4,6 days after myoblast differentiation. (C) Quantitative assay for Ezrinexpressionswas analyzed 2, 4 and 6 days after myoblast differentiation. $n=3, * P \otimes 0.05$ vs. 0 day; \#Pख0.05 vs. 2 day; \&P®0.05 vs. 4 day. (E)Typical image of MyHC staining in differentiated $\mathrm{C} 2 \mathrm{C} 12$ myoblast cells treated by overexpression or knockdown of Ezrin for 2, 46 days. (F-H)Quantitative assay for the number of MyHC+ myotubes with more than 5 nuclei was analyzed 2, 4 and 6 days after myoblast differentiation treated by overexpression or knockdown of Ezrin, respectively. $n=3, * P \otimes 0.05$ vs. Ctrl; \#Pख0.05 vs. Ctrl. 


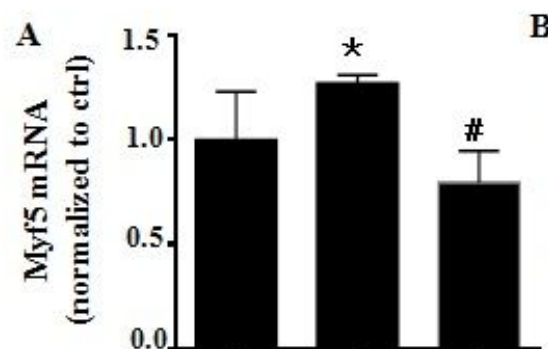

Ad-Ezrin

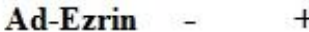

Ad-shEzrin

D

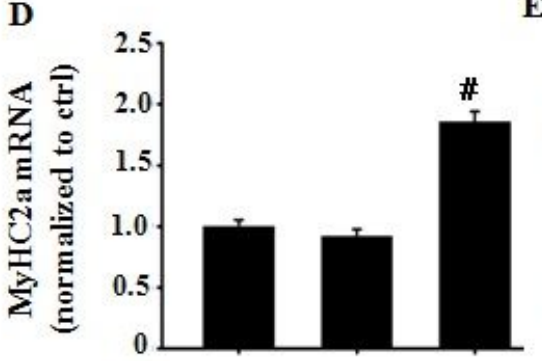

Ad-Ezrin

Ad-shEzrin

G

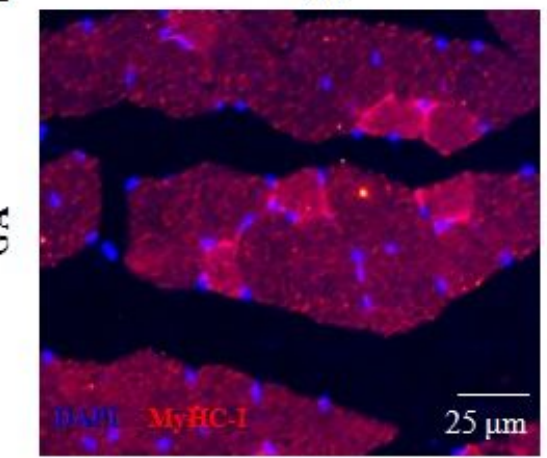

I

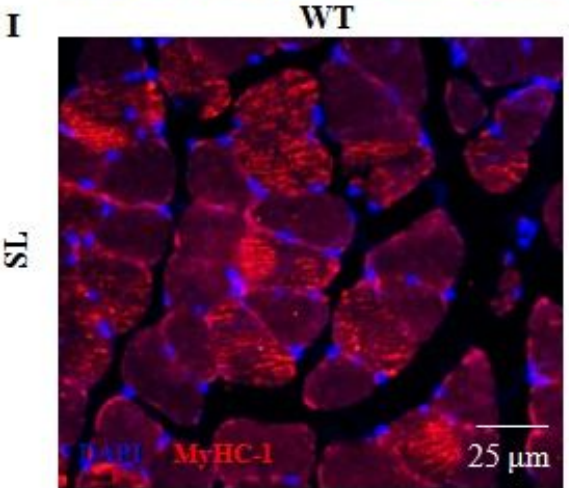

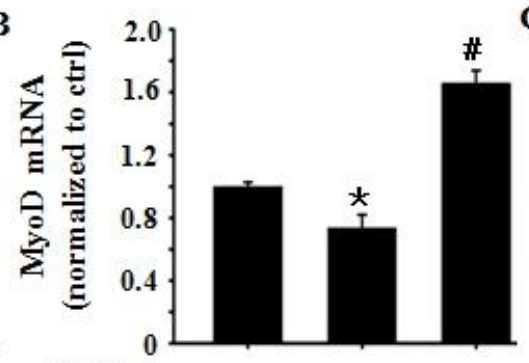

Ad-Ezrin

Ad-shEzrin

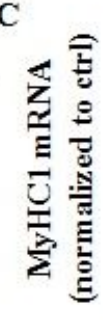

Ad-Ezrin

Ad-shEzrin
E

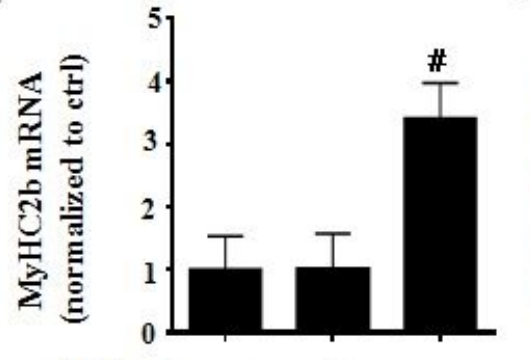

Ad-Ezrin
吾

Ad-Ezrin

Ad-shEzrin

H

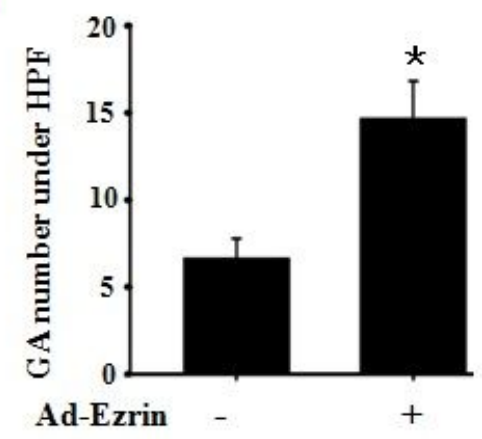

Ad-Ezrin
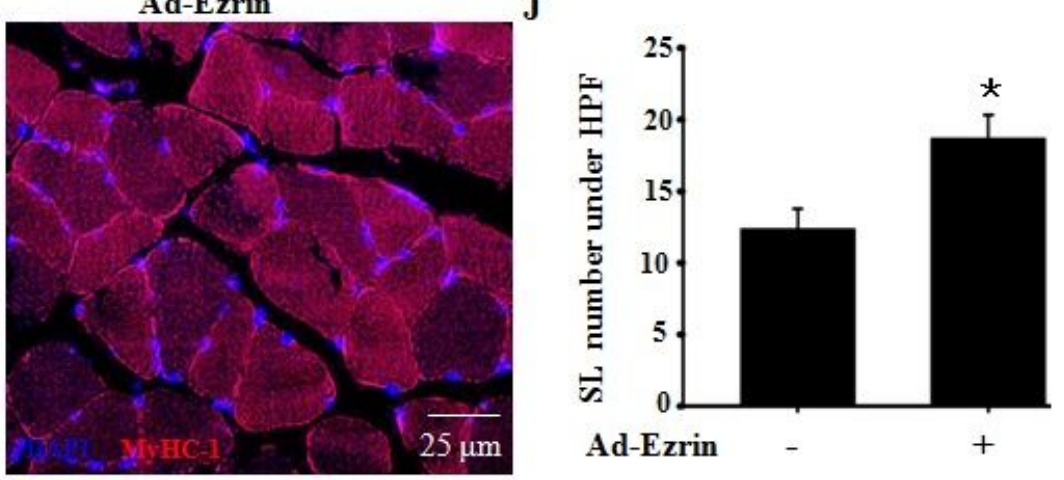

Figure 2

Ezrin altered the muscle fiber types. (A-B) Myf5 and MyoD, myoblast cells differentiation regulatory factors, were altered in differentiated $\mathrm{C} 2 \mathrm{C} 12$ cells pretreated with Ad-AdEzrin or Ad-shEzrin. (C) MyHC1, as one of type I muscle fiber maker, were repressed in differentiated $\mathrm{C} 2 \mathrm{C} 12$ cells exposed to overexpression or knockdown of Ezrin by detecting the levels of mRNA using Real-time PCR. (D-F) Overexpression of Ezrin promoted MyHC-1 expressions while knockdown of Ezrin increased the levels of type II muscle fiber 
makers such as MyHC-2a and MyHC-2b in differentiated C2C12 cellspretreated with Ad-AdEzrin or AdshEzrinby detecting the levels of mRNA using Real-time PCR. n=3, *Pヌ0.05 vs. Ctrl; \#Pヌ0.05 vs. Ctrl. (G-J) Iv viv assay for MyHC-1 expressions in gastrocnemius (GA) and musculi soleus (SL) following the local injection of Ad-Ezrin. $n=6$, *P®0.05 vs. WT.

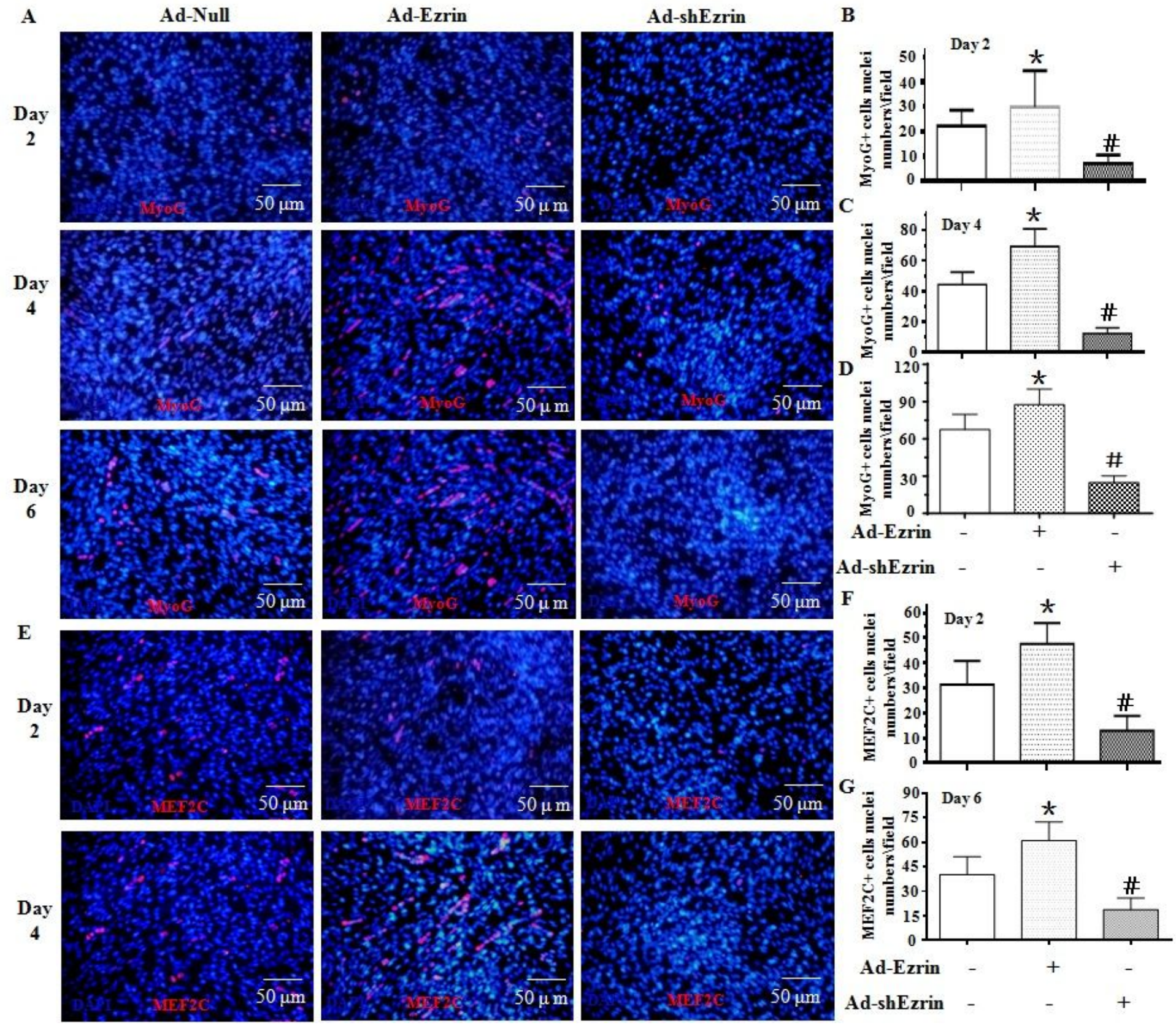

Figure 3

MyoG/MEF2C involved Ezrin-mediated myoblast differentiation and fusion. (A) Typical image of MyoGstaining in differentiated C2C12 myoblast cells treated by overexpression or knockdown of Ezrin for 2, 46 days. (B-D)Quantitative assay for the number of MyoG+ nucleiwas analyzed 2, 4 and 6 days after myoblast differentiation in treated by overexpression or knockdown of Ezrin, respectively. $n=3, * P \otimes 0.05$ vs. Ctrl; \#P『0.05 vs. Ctrl.(E-G)Typical image of MEF2c staining in differentiated C2C12 myoblast cells treated by overexpression or knockdown of Ezrin for 2, 46 days.Quantitative assay for the number of MEF2c+ 
nuclei was analyzed 2, 4 and 6 days after myoblast differentiation in treated by overexpression or knockdown of Ezrin, respectively. $n=3$, *Pख0.05 vs. Ctrl; \#Pख0.05 vs. Ctrl.
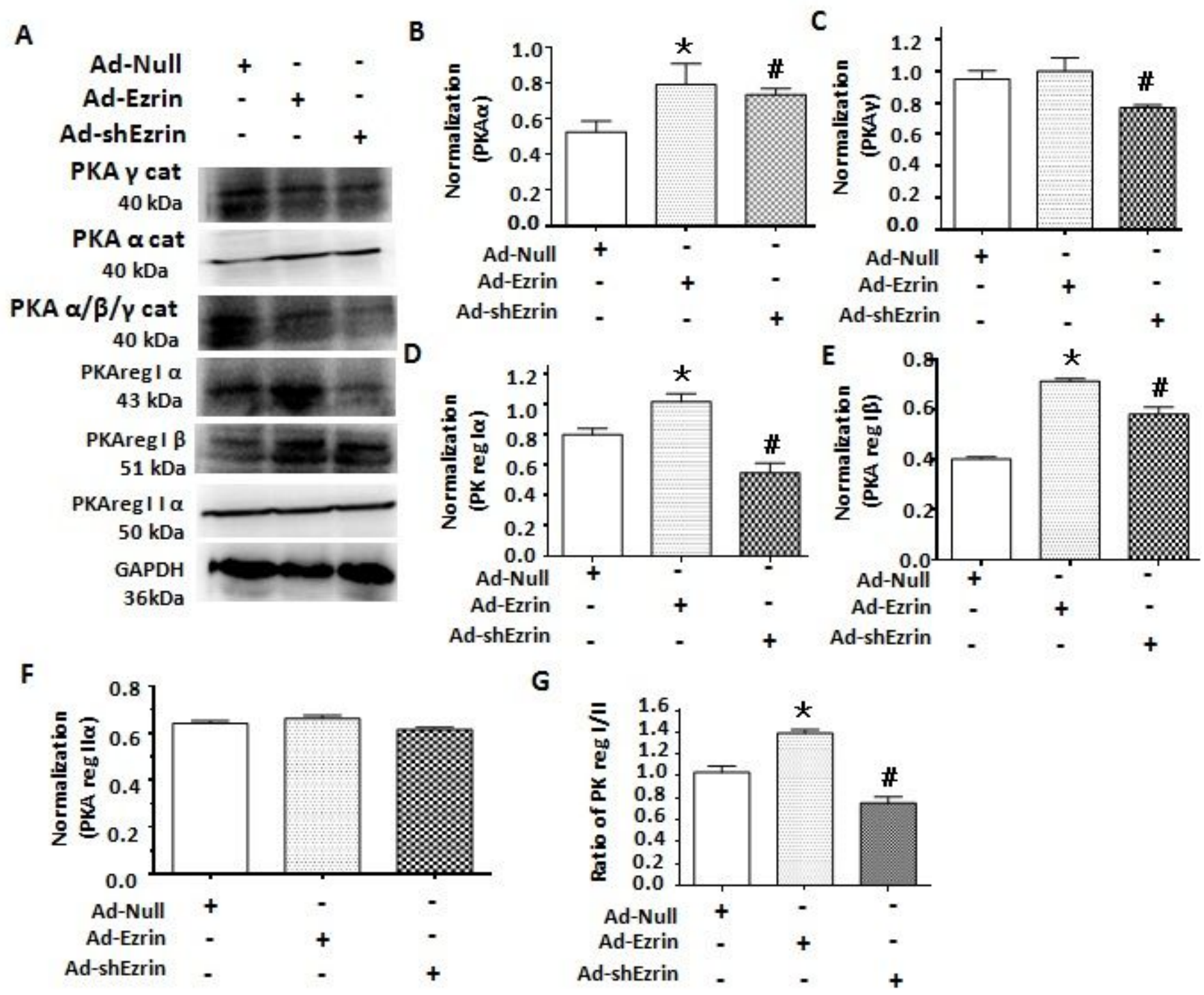

Figure 4

Ezrin regulated myoblast differentiation and fusion through PKA signaling pathway. (A) Western blot for indicated proteins in myoblast cells treated by over-expressionor knockdown of Ezrin for 4 days. (B-G) Quantitative assay for indicated proteins was analyzed4 days after myoblast differentiation following the treatment of over-expressionor knockdown of Ezrin. $n=3, * P \llbracket 0.05$ vs. Ctrl;\#Pख0.05 vs. Ctrl. 


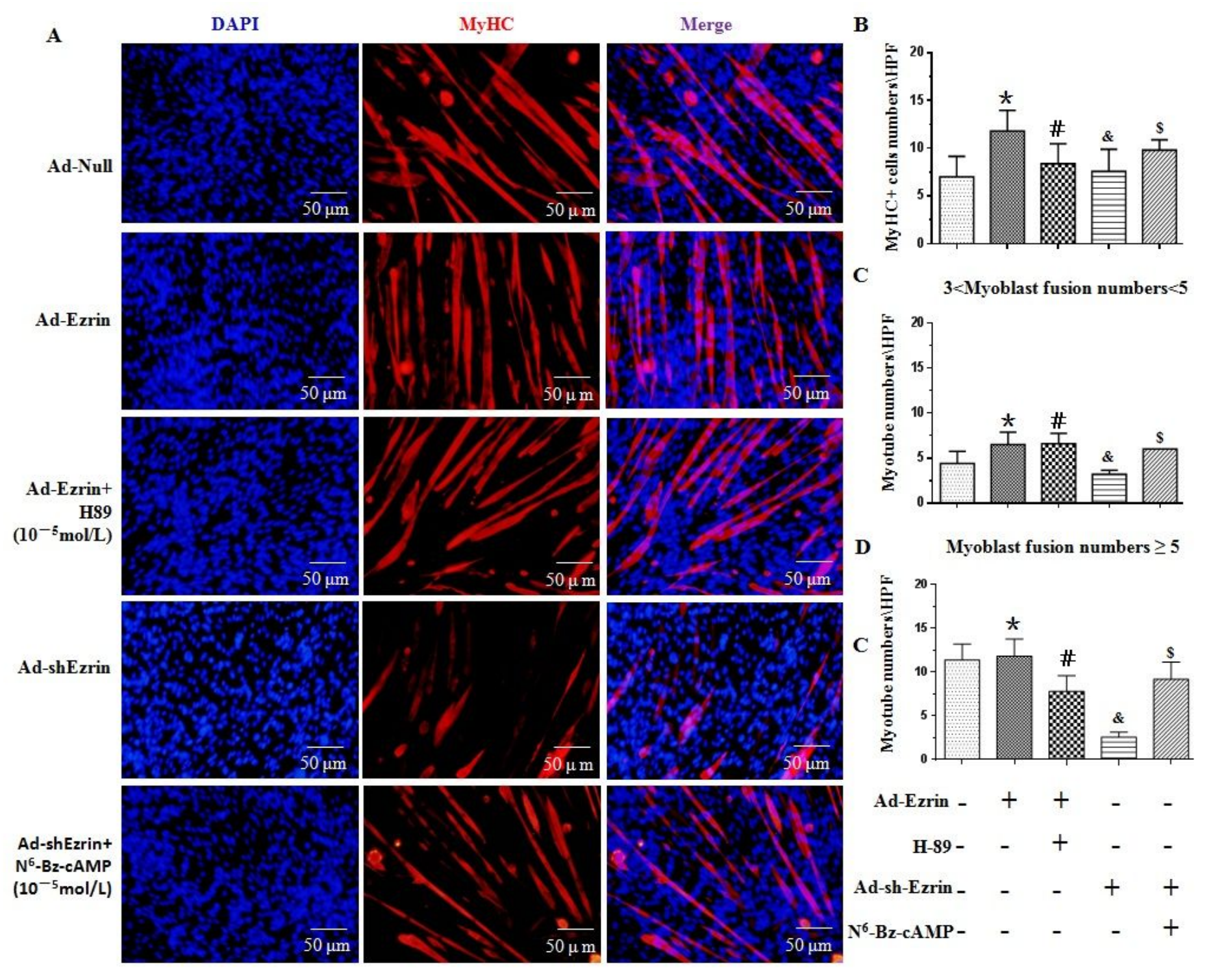

\section{Figure 5}

PKA involved Ezrin-mediated myoblast differentiation and fusion. (A) Typical image of MyHCstaining in differentiated $\mathrm{C} 2 \mathrm{C} 12$ myoblast cells treated by Ad-Ezrin with or without $\mathrm{H}-89(10-5 \mathrm{~mol} / \mathrm{L})$, or Ad-shEzrin with or without N6-Bz-CAMP(10-5mol/L).(B-D)Quantitative assay for the number of MyHC+ myotubes with 3-5 or more than 5 nuclei were analyzed six days after myoblast differentiation. $n=3$, *P凶0.05 vs. Ctrl; $\# P \llbracket 0.05$ vs. Ad-Ezrin; \&P囚0.05 vs. Ctrl; $\$ P \rrbracket 0.05$ vs. Ad-shEzrin. 
A

Ad-Null

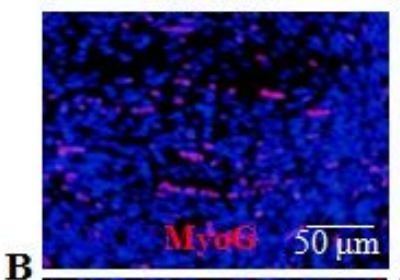

B

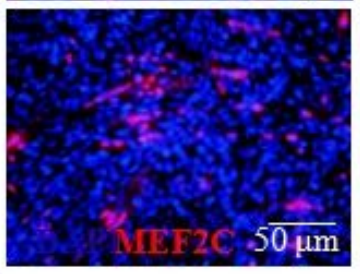

C

D
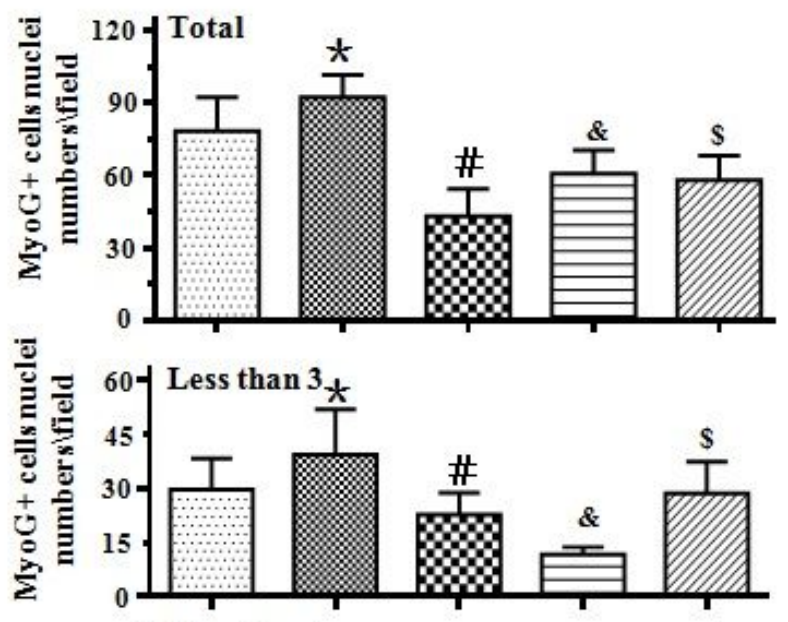

$\mathbf{E}$

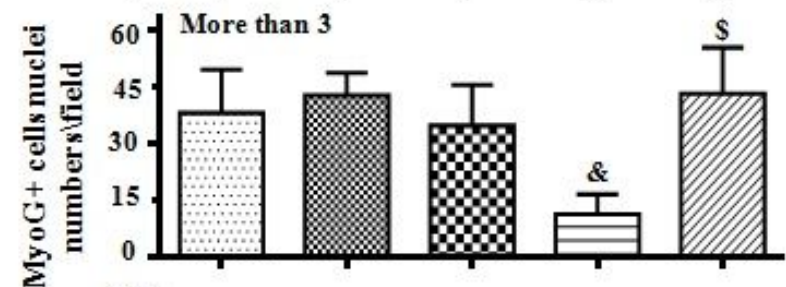

F

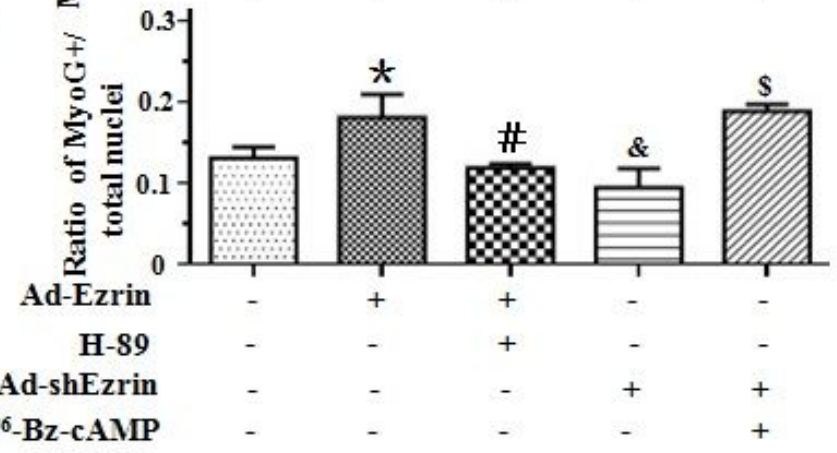

Ad-Ezrint
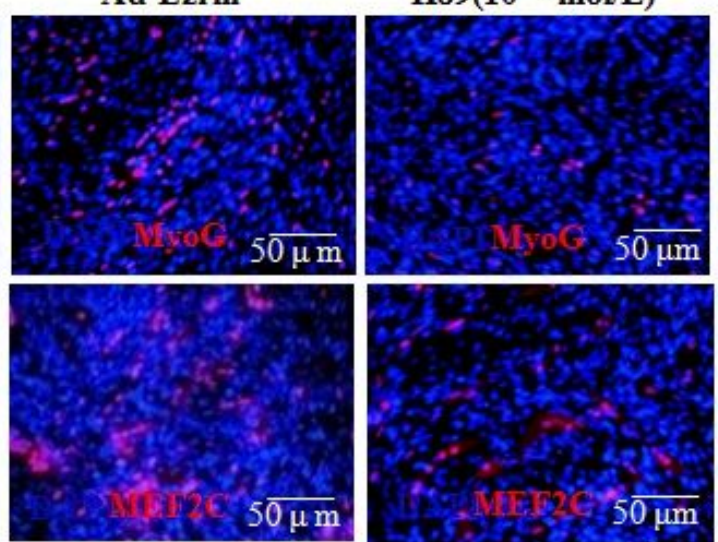

Ad-shEzrin $+N^{6}-B z-$

$\operatorname{cAMP}\left(10^{-5} \mathrm{~mol} / \mathrm{L}\right)$
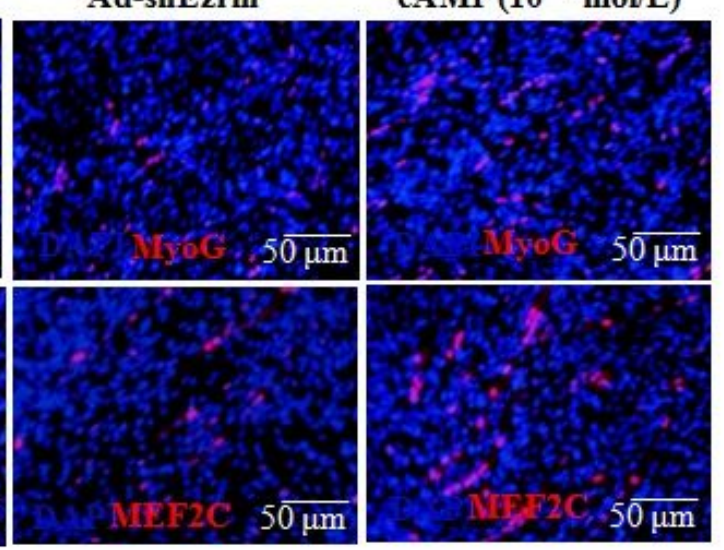

G

H

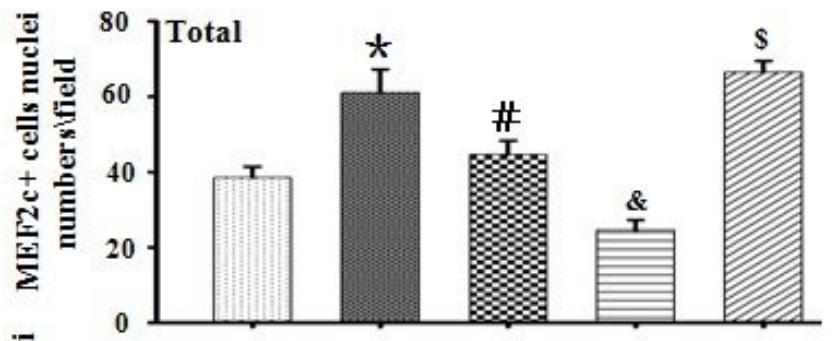

I

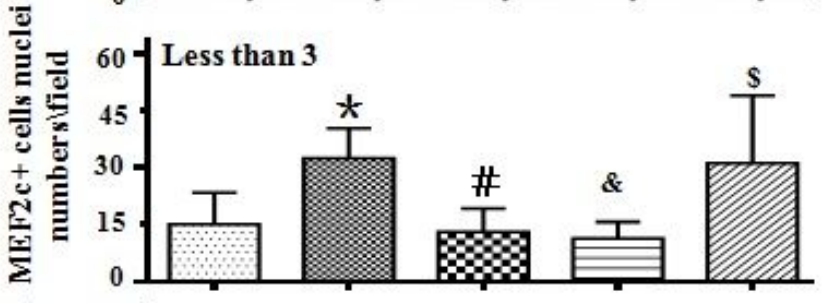

I

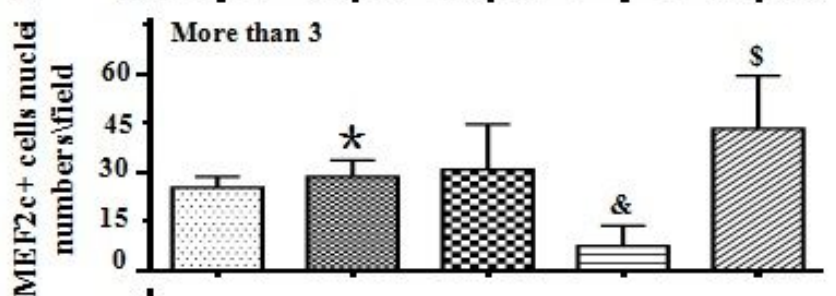

$\mathbf{J}$

J

Ad-Ezrin

H-89

Ad-shEzrin

No-Bz-cAMP

\section{Figure 6}

Ezrin regulated myoblast differentiation and fusion through PKA-MyoG/MEF2C signaling pathway. (A-

B)Typical image of MyoG and MEF2cstaining in differentiated C2C12 myoblast cells treated by Ad-Ezrin with or without H-89(10-5mol/L), or Ad-shEzrin with or without N6-Bz-CAMP(10-5mol/L), respectively. (CJ)Quantitative assay for the number of MyoG+ or MEF2c+ nuclei was analyzed 6 days after myoblast 
differentiation treated by Ad-Ezrin with or without H-89, or Ad-shEzrin with or without N6-Bz-cAMP(10$5 \mathrm{~mol} /$ L. n=3, *P囚0.05 vs. Ctrl; \#Pख0.05 vs. Ad-Ezrin; \&Pख0.05 vs. Ctrl; \$Pख0.05 vs. Ad-shEzrin.

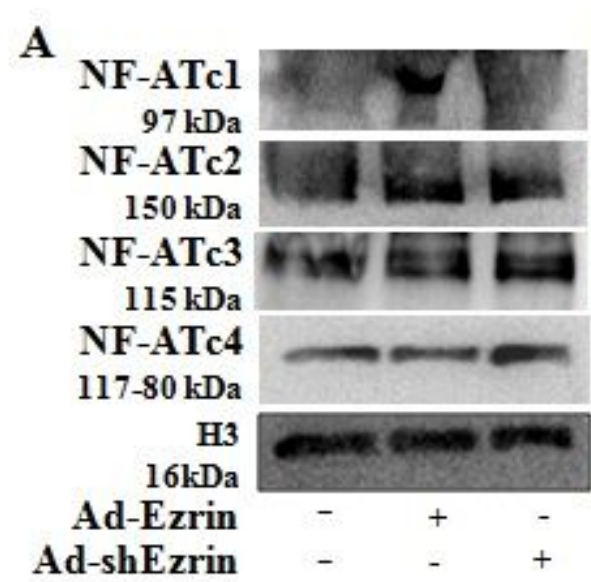

D

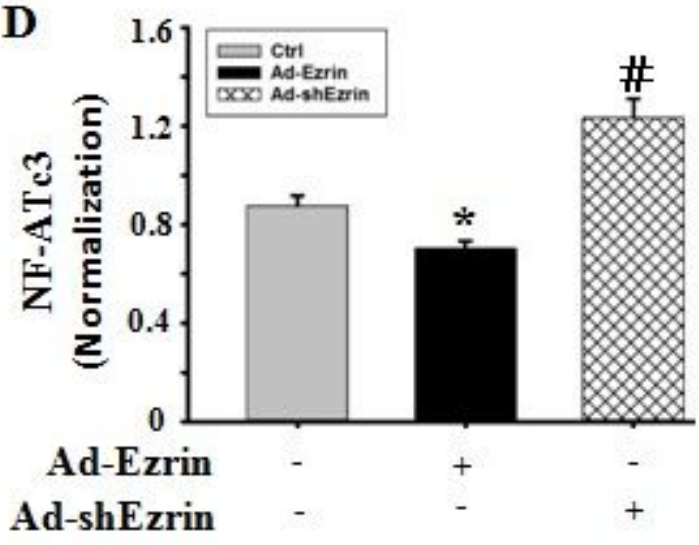
$\begin{array}{rrrr}\underset{A d-E z r i n}{\text { Ad-shEzrin }} & - & + & -\end{array}$
B

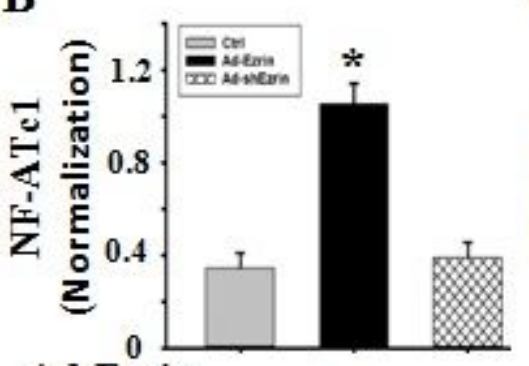

Ad-Ezrin - + Ad-shEzrin - - +

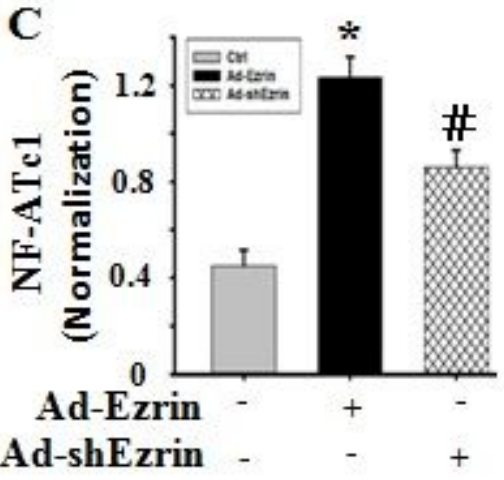

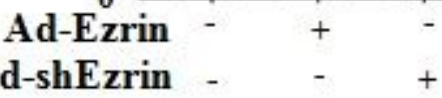

$\mathbf{E}$

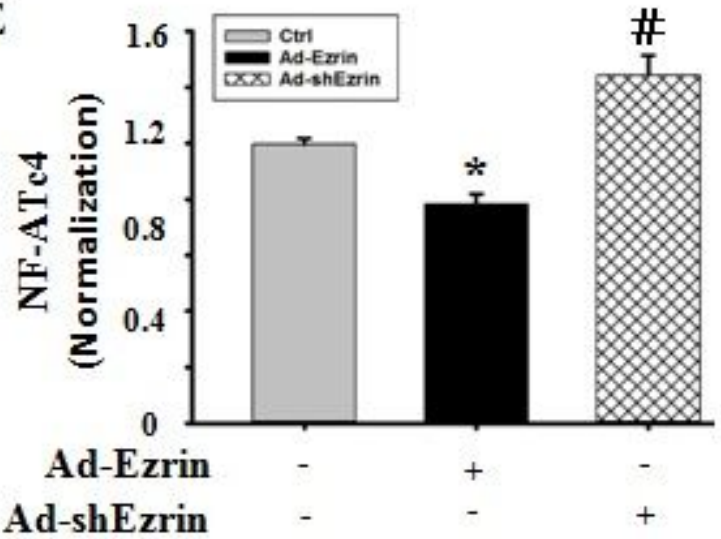

Figure 7

NFATs involved in Ezrin-mediated myoblast differentiation and fusion. (A)Western blot for indicated proteins in myoblast cells treated by over-expressionor knockdown of Ezrin for 4 days. (B-E) Quantitative assay for indicated proteins was analyzed 4 days after myoblast differentiation following the treatment of over-expressionor knockdown of Ezrin. $n=3$, *P囚0.05 vs. Ctrl;\#Pख0.05 vs. Ctrl. 


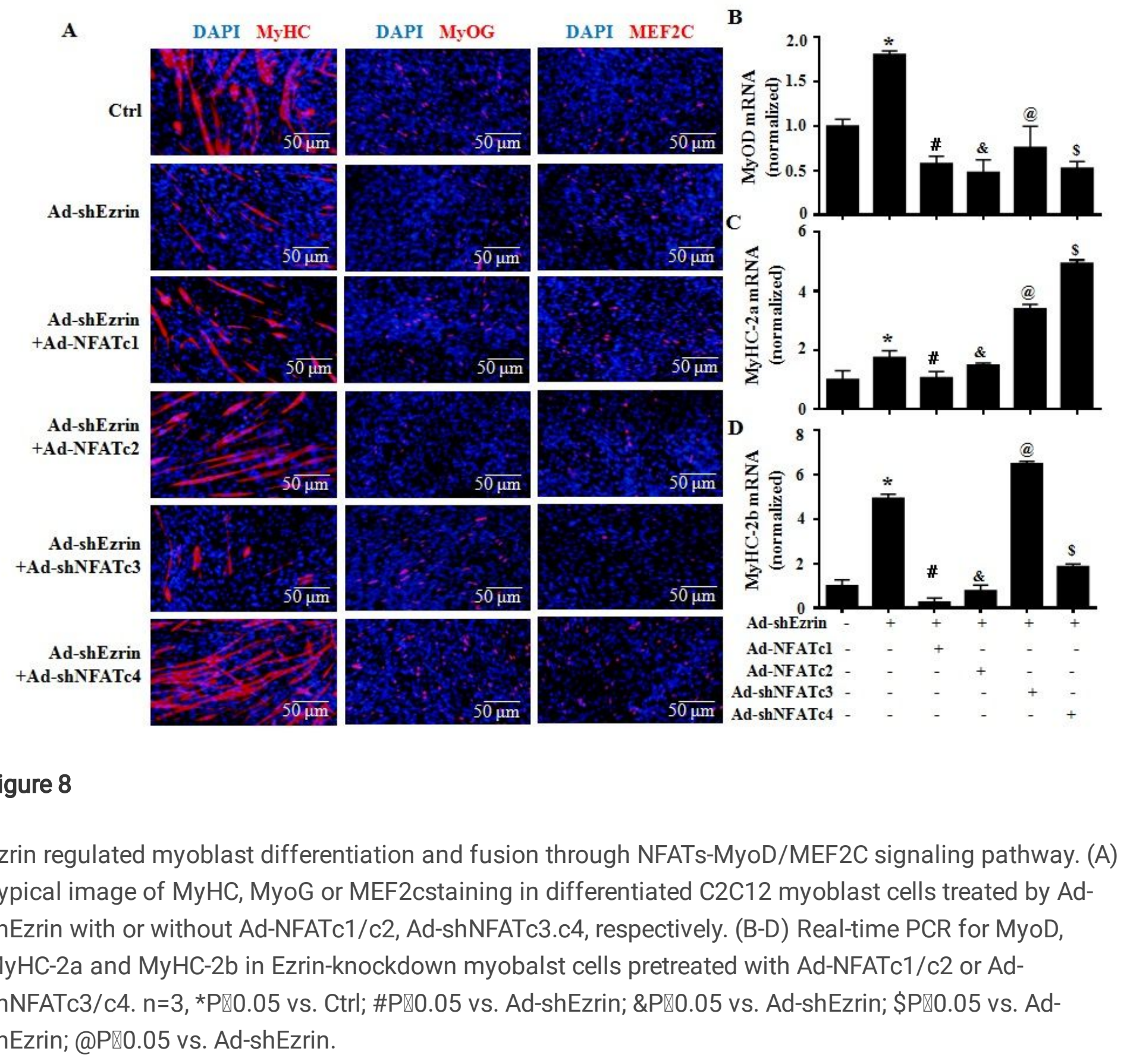




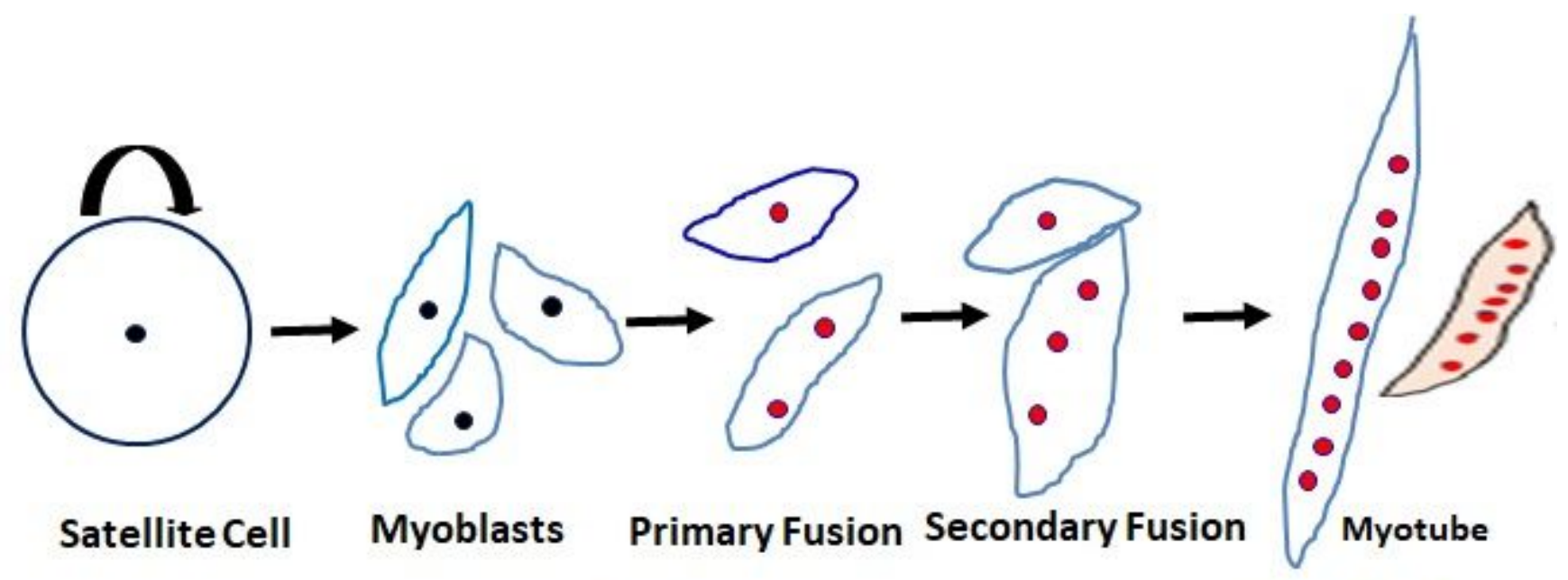

\section{Ezrin}

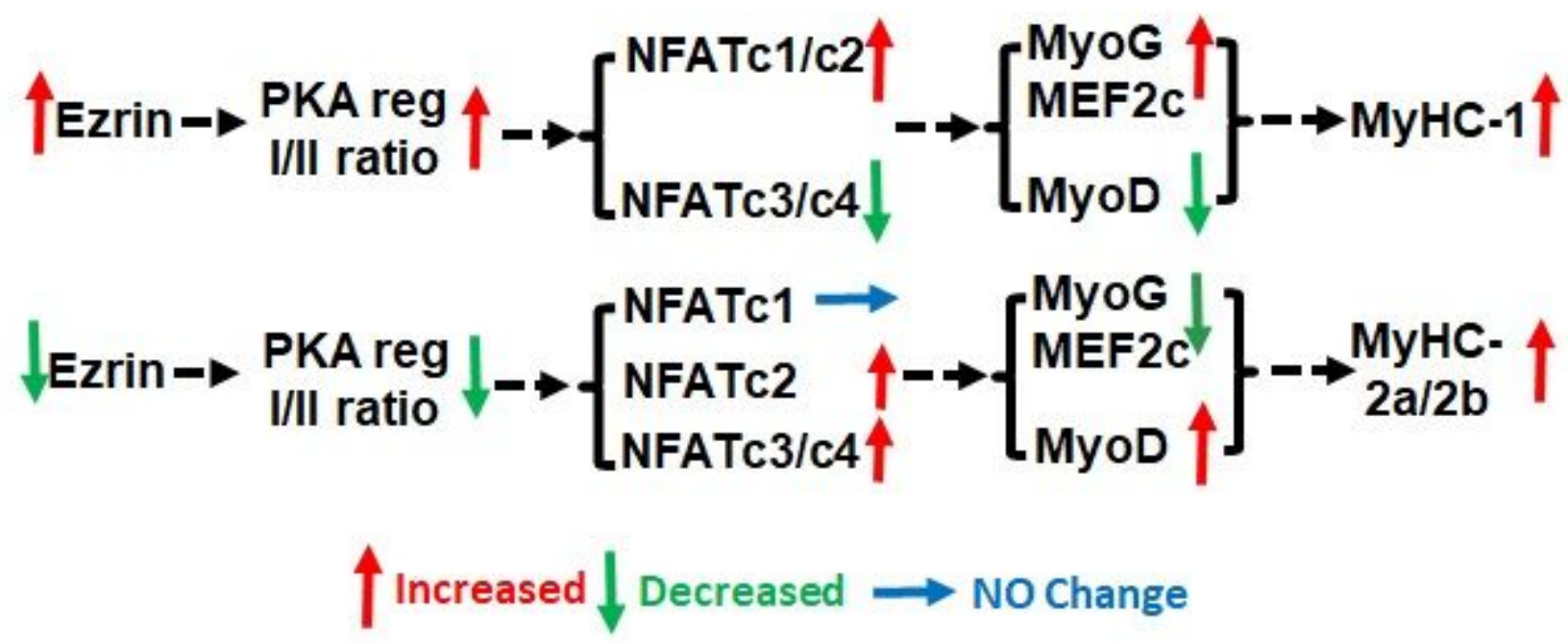

Figure 9

Working Model: Ezrin regulated myoblast differentiation/fusion and muscle fiber specializationthrough PKA-NFAT-MyoD/MEF2csignalling pathway. Ezrin expression show a time-dynamic characteristic during myoblast differentiation and fusion. Ezrin promoted myoblast differentiation and fusion through altering PKA RI/RII ratio, triggering type I muscle fiber specialization through NFATc2-MEF2C signaling pathway.Knockdown of Ezrin inhibited myoblast differentiation/fusion, especially type llb muscle fiber specialization through NFATc4-MyoD/MEF2C signaling pathway.

\section{Supplementary Files}

This is a list of supplementary files associated with this preprint. Click to download.

- Supplemental.docx 
- sFigure1.tif

- sFigure2.tif

- sFigure3.tif

Page 26/26 